Check for updates

Cite this: RSC Adv., 2019, 9, 31543

Received 9th May 2019

Accepted 23rd September 2019

DOI: 10.1039/c9ra03482j

rsc.li/rsc-advances

\title{
Laser cooling of InF, InCl and InH with an ab initio study
}

\author{
Rong Yang, (D) *a Bin Tang ${ }^{\mathrm{b}}$ and XiangYu Han ${ }^{\text {ac }}$
}

The feasibility of laser cooling $\mathrm{InF}, \mathrm{InCl}$ and $\mathrm{InH}$ is investigated based on ab initio quantum chemistry. To determine their suitability for laser cooling molecules, we have calculated the electronic structures, spectroscopic parameters, transition dipole moments (TDMs), radiative lifetimes, Franck-Condon factors (FCFs) and diode laser excitation wavelengths of $\mathrm{InF}, \mathrm{InCl}$ and $\mathrm{InH}$. Calculated spectroscopic constants of the first three electronic states for $\mathrm{InF}, \mathrm{InCl}$ and $\mathrm{InH}$ show good agreement with available theoretical and experimental results. InF has highly diagonally distributed FCFs $\left(f_{00}=0.961, f_{11}=0.909\right)$ for the $C^{1} \Pi \rightarrow$ $X^{1} \Sigma^{+}$transition, and the rather short lifetime of the state $C^{1} \Pi$ is computed to be $2.77 \mathrm{~ns}$ at the lowest vibrational level. Notable is that the ${ }^{3} \Pi \rightarrow X^{1} \Sigma^{+}$transition of InF also has large diagonal FCFs and short lifetimes. Therefore, $\mathrm{InCl}$ and $\mathrm{InH}$ are not potential laser-cooling candidates because the FCFs of the ${ }^{1} \Pi$ $\rightarrow \mathrm{X}^{1} \Sigma^{+}$transition are off-diagonal. We further propose laser cooling schemes for InF. The present results could provide a promising theoretical reference for further theoretical and experimental research on $\mathrm{InF}, \mathrm{InCl}$ and $\mathrm{InH}$.

\section{Introduction}

The production of ultracold diatomic molecules has drawn considerable interest for several years because of their wide range of prospective applications, for example, quantum computations, ${ }^{1}$ controlling chemistry ${ }^{2}$ and precision measurement. ${ }^{3-5}$ Recently, laser cooling of diatomic molecules $\mathrm{SrF}^{6,7}$ $\mathrm{YO}^{8}{ }^{8} \mathrm{CaF}^{9}$ and $\mathrm{KRb}^{10}$ have been experimentally performed. These successful laser cooling experiments have initiated a search for more molecules since more than 90 elements in the periodic table possibly form more than 4000 different diatomic molecules. The potential laser cooling candidates must have highly diagonal Franck-Condon factors (FCFs) and shorter lifetimes (i.e. high spontaneous-emission rates). Highly diagonal FCFs limit the number of lasers required to keep the molecule in a quasi-closed-loop cooling cycle and rapid laser cooling demands a shorter lifetime.

The $A^{2} \Pi \rightarrow X^{2} \Sigma^{+}$transition suggested by the experiments ${ }^{6-9}$ for SrF, YO and $\mathrm{CaF}$ has been theoretically used to establish the laser cooling schemes for $\mathrm{RaF},{ }^{4} \mathrm{BeF},{ }^{11} \mathrm{MgF}^{12}$ and $\mathrm{MH}^{13}(\mathrm{M}=\mathrm{Be}$, $\mathrm{Mg}$, Ca, Sr, and Ba). In 2015, laser cooling of LiBe molecule based on the $\mathrm{B}^{2} \Pi \rightarrow \mathrm{X}^{2} \Sigma^{+}$transition has been reported by You et al. ${ }^{14}$ Interestingly, $\mathrm{AlX}\left(\mathrm{X}=\mathrm{H},{ }^{15} \mathrm{~F},{ }^{15} \mathrm{Cl}^{16}\right.$ and $\left.\mathrm{Br}^{16}\right)$ and $\mathrm{BBr}^{17} /$ $\mathrm{BCl}^{17}$ molecules with an intervening state $\mathrm{a}^{3} \Pi$ existed between

${ }^{a}$ School of Mathematics and Physics, Chongqing Jiaotong University, Chongqing 400074, PR China.E-mail: cqyr88@126.com

${ }^{b}$ Institute of Finance \& Trade, Chongqing City Management College, Chongqing 401331, PR China

'Institute of Atomic and Molecular Physics, Sichuan University, Chengdu 610065, PR China the $A^{1} \Pi$ and $X^{1} \Sigma^{+}$states have also been identified as potential laser-cooling candidates. The laser cooling scheme using the $\mathrm{A}^{1} \Pi \rightarrow \mathrm{X}^{1} \Sigma^{+}$and $\mathrm{a}^{3} \Pi \rightarrow \mathrm{X}^{1} \Sigma^{+}$transitions was theoretically suggested. In our previous works, theoretical optical schemes have been predicted for $\mathrm{AlCl}^{16} / \mathrm{AlBr}^{16}$ and $\mathrm{BBr}^{17} / \mathrm{BCl}^{17}$ Li et al. ${ }^{18}$ propose to utilize the ${ }^{1} \Sigma-{ }^{1} \Sigma$ electronic transition system for direct laser cooling of $\mathrm{AgH}$ and $\mathrm{AgD}$ molecules.

The boron group molecules are of persistent interest in the investigation of cold molecules. $\mathrm{BH}^{19} / \mathrm{BCl}^{17} / \mathrm{BBr},{ }^{17} \mathrm{AlH}^{15} / \mathrm{AlF}^{15} /$ $\mathrm{AlCl}^{16} / \mathrm{AlBr}^{16}$ and $\mathrm{GaF}^{19}$ were identified as the promising lasercooling targets theoretically. However, the study on laser cooling of indium diatomics is very limited. On the basis of previous studies, we believe InF, InCl and InH molecules are possible laser-cooling candidates. In this paper, we focus on the theoretical studies of the laser cooling of InF, InCl and InH. The electronic structure and spectroscopic properties of the lowlying electronic states of $\mathrm{InF}$ were given by Banerjee et al. ${ }^{20}$ from the relativistic configuration interaction calculations. They also determined the lifetime of the state $\mathrm{C}^{1} \Pi$ was about 2.3 ns at the lowest vibrational level. Zou et al. ${ }^{21}$ obtained the potential energy curves and the spectroscopic constants on the ground and low-lying excited states of InCl using all-electron relativistic calculations. The theoretical calculations have been performed on the InH molecule based on multi-reference configuration interaction plus Davidson corrections method. ${ }^{22}$ Leininger $e t a l .^{23}$ performed the calculations for spectroscopic constants of $\mathrm{InH}, \mathrm{InF}$, and $\mathrm{InCl}$ at the self-consistent-field and correlated levels. Several experimental results on the spectroscopic parameters were available in the literature for $\mathrm{InF}$, InCl and InH. 
Though InF, InCl and InH have been extensively investigated in previous works, as mentioned above, a systematic study of laser cooling of InF, InCl and InH is not available in the literature to the best of our knowledge. The main objective of this paper is to identify whether $\mathrm{InF}$, InCl and $\mathrm{InH}$ are potential laser-cooling candidates and briefly design laser-cooling schemes. To determine their suitability for laser cooling molecules, we have calculated the electronic structures, spectroscopic parameters, TDMs, radiative lifetimes, FCFs and diode laser excitation wavelengths of $\mathrm{InF}$, InCl and $\mathrm{InH}$. Since in this work we mean to focus on the vibrational cooling, the rotational degrees of freedom can be neglected in the first approximation. Here, the $\mathrm{C}^{1} \Pi \rightarrow \mathrm{X}^{1} \Sigma^{+}$transition for $\mathrm{InF} / \mathrm{InCl}$ and $\mathrm{A}^{1} \Pi \rightarrow \mathrm{X}^{1} \Sigma^{+}$ transition for InH are the main transitions of laser cooling and the transition from intervening state ${ }^{3} \Pi$ to the ground state $\mathrm{X}^{1} \Sigma^{+}$is spin forbidden.

The paper is organized as follows. Section 2 details the theoretical methods and basis sets used in the calculations. Section 3 presents the results and discussions of the data, outlining the schemes for direct laser cooling. Section 4 provides a conclusion for this work.

\section{Computational details}

All the $a b$ initio calculations are performed with the MOLPRO package. ${ }^{24}$ It should be noted that only the subgroup $C_{2 \mathrm{v}}$ point group symmetry can be adopted for InF, InCl and InH in the package. The electronic structures for the $\mathrm{X}^{1} \Sigma^{+}, \mathrm{C}^{1} \Pi$ and ${ }^{3} \Pi$ states of $\mathrm{InF} / \mathrm{InCl}$ and the $\mathrm{X}^{1} \Sigma^{+}, \mathrm{A}^{1} \Pi$ and $\mathrm{a}^{3} \Pi$ states of $\mathrm{InH}$ are calculated using the complete active space self-consistent field method (CASSCF) ${ }^{25,26}$ which is followed by MRCI plus Davidson corrections (MRCI+Q) ${ }^{27-29}$ calculations. The scalar relativistic effect is taken into account using the Douglas-Kroll-Hess (DHK) ${ }^{30,31}$ transformation of the relativistic Hamiltonian. The spectroscopic constants, including equilibrium bond distance $\left(R_{\mathrm{e}}\right)$, dissociation energy $\left(D_{\mathrm{e}}\right)$, harmonic frequency $\left(w_{\mathrm{e}}\right)$, anharmonic vibrational frequency $\left(w_{\mathrm{e}} \chi_{\mathrm{e}}\right)$, rotational constant $\left(B_{\mathrm{e}}\right)$ and electronic transition energy $\left(T_{\mathrm{e}}\right)$ are evaluated by LEVEL 8.0 program. ${ }^{32}$ The TDMs of InF, InCl and InH are computed by taking the expectation and transition values using the MRCI wave functions. With the potential energy curves (PECs) and TDMs of different electronic states, we have obtained the FCFs and radiative lifetimes of the various vibrational levels from LEVEL 8.0 program.

For the indium atom, being considerably heavy, we use the small-core scalar relativistic effective core potential ECP28MDF ${ }^{33}$ together with the corresponding valence basis sets. The $4 \mathrm{~s}^{2} 4 \mathrm{p}^{6} 4 \mathrm{~d}^{10} 5 \mathrm{~s}^{2} 5 \mathrm{p}^{1}$ electrons of the In atom are kept in the valence space, while the remaining core electrons are replaced by the above mentioned pseudopotentials. The correlation consistent polarized valence quintuple zeta aug-cc-pV5Z $(=\mathrm{AV} 5 \mathrm{Z})^{34}$ is chosen for $\mathrm{H}$. Meanwhile, the AVQZ ${ }^{35}$ all-electron basis sets are employed for F and Cl. That is to say, for InF, nineteen molecule orbitals (MOs) are put into active space, including $9 \mathrm{a}_{1}, 4 \mathrm{~b}_{1}, 4 \mathrm{~b}_{2}, 1 \mathrm{a}_{2}$ symmetry $(9,4,4,1)$, which correspond to the 1s2s2p shells of the F atom and 4s4p4d5s5p shells of the In atom; for InCl, twenty two MOs are selected as the active space, including $11 a_{1}, 5 b_{1}, 5 b_{2}, 1 a_{2}$ symmetry $(11,5,5,1)$, which correspond to the $\mathrm{Cl} 1 \mathrm{~s} 2 \mathrm{~s} 2 \mathrm{p} 3 \mathrm{~s} 3 \mathrm{p}$ and In $4 \mathrm{~s} 4 \mathrm{p} 4 \mathrm{~d} 5 \mathrm{~s} 5 \mathrm{p}$ shells; for InH, the active space is $(7,3,3,1)$, which correspond with the $\mathrm{H}$ 1s and In 4s4p4d5s5p.

The main transitions of laser cooling for $\mathrm{InF}$, InCl and $\mathrm{InH}$ are the ${ }^{1} \Pi \rightarrow \mathrm{X}^{1} \Sigma^{+}$transitions which are singlet states. Moreover, the excited state decays exclusively on the ${ }^{1} \Pi \rightarrow \mathrm{X}^{1} \Sigma^{+}$ transition because of the selection rules for the change in parity and angular momentum in an electric dipole transition. Besides, the transition from intervening state ${ }^{3} \Pi$ to the ground state $\mathrm{X}^{1} \Sigma^{+}$is spin forbidden. Therefore, spin-orbit coupling effects are not considered in the calculations. Previous works on laser cooling of $\mathrm{MH}^{13}(\mathrm{M}=\mathrm{Be}, \mathrm{Mg}, \mathrm{Ca}, \mathrm{Sr}$, and $\mathrm{Ba})$ and $\mathrm{GaF}^{19}$ also indicate that the influence of spin-orbit coupling on the spectroscopic properties is generally weak.

\section{Results and discussions}

\subsection{PECs and spectroscopic constants}

The calculated PECs of $\mathrm{X}^{1} \Sigma^{+},{ }^{1} \Pi$ and ${ }^{3} \Pi$ states of InF, InCl and InH acquired at the MRCI level are shown in Fig. 1. The PECs of $\mathrm{X}^{1} \Sigma^{+}$and ${ }^{3} \Pi$ states look surprisingly similar, implying that highly diagonal FCFs for ${ }^{3} \Pi \rightarrow \mathrm{X}^{1} \Sigma^{+}$transitions are possible. In Table 1, we report the corresponding spectroscopic constants of $\mathrm{InF} / \mathrm{InCl}$ for the $\mathrm{X}^{1} \Sigma^{+}, \mathrm{C}^{1} \Pi$ and ${ }^{3} \Pi$ states and InH for the $\mathrm{X}^{1} \Sigma^{+}$, $\mathrm{A}^{1} \Pi$ and $\mathrm{a}^{3} \Pi$ states. The experimental ${ }^{36}$ and theoretical ${ }^{20-23}$ values for these states are also listed in Table 1 for comparison.

For the ground state $\mathrm{X}^{1} \Sigma^{+}$of $\mathrm{InF}$, the calculated $R_{\mathrm{e}}$ and $w_{\mathrm{e}}$ results are only $0.002 \AA$ and $14.69 \mathrm{~cm}^{-1}$ larger, while the $D_{\mathrm{e}}$ result is only $0.03 \mathrm{~cm}^{-1}$ smaller than the experimental data. ${ }^{36}$ The corresponding percentage errors in $R_{\mathrm{e}}, w_{\mathrm{e}}$ and $D_{\mathrm{e}}$ are $0.10 \%$, $3.08 \%$ and $0.55 \%$, respectively. Banerjee et al. ${ }^{20}$ provided even larger $R_{\mathrm{e}}(1.26 \%)$ and $w_{\mathrm{e}}(5.35 \%)$, an even smaller $D_{\mathrm{e}}(2.00 \%)$ for the $\mathrm{X}^{1} \Sigma^{+}$of InF compared to the experimental data. ${ }^{36}$ The first excited state of InF is ${ }^{3} \Pi$ which lies well above the ground state. Our calculated $R_{\mathrm{e}}$ and $w_{\mathrm{e}}$ of the ${ }^{3} \Pi$ state are in remarkably good agreement with experiment, ${ }^{36}$ and the relative errors are only $0.36 \%$ and $2.38 \%$, respectively. While the percentage errors in $R_{\mathrm{e}}$ and $w_{\mathrm{e}}$ given by Banerjee et al. ${ }^{20}$ are $1.74 \%$ and $2.56 \%$, respectively. The present calculation yields a $T_{\mathrm{e}}$ value for the ${ }^{3} \Pi$ of $30463 \mathrm{~cm}^{-1}$, and the observed $\mathrm{A}^{3} \Pi_{0^{+}}, \mathrm{B}^{3} \Pi_{1}-\mathrm{X}^{1} \Sigma^{+}$transitions $^{36}$ take place in the range $30400-31300 \mathrm{~cm}^{-1}$. The relativistic configuration interaction calculations by Banerjee $e$ t $a l^{20}$ yielded a $T_{\mathrm{e}}$ value for the ${ }^{3} \Pi$ of $28145 \mathrm{~cm}^{-1}$ which was not within the observed range. It is also encouraging to see that the present values of $R_{\mathrm{e}}, w_{\mathrm{e}}$ and $T_{\mathrm{e}}$ for the $\mathrm{C}^{1} \Pi$ state are $1.964 \AA$, $467.05 \mathrm{~cm}^{-1}$ and $43050 \mathrm{~cm}^{-1}$, which are in very good agreement with the experimental data $1.966 \AA, 463.90 \mathrm{~cm}^{-1}$ and $42809 \mathrm{~cm}^{-1}$ and the theoretical data $2000 \AA, 460.00 \mathrm{~cm}^{-1}$ and $42255 \mathrm{~cm}^{-1}$.

For InCl, good agreement is found between our results and experimental data. The percentage errors of $R_{\mathrm{e}}, w_{\mathrm{e}}$ and $D_{\mathrm{e}}$ for the ground state $\mathrm{X}^{1} \Sigma^{+}$are $0.12 \%, 2.56 \%$ and $0.43 \%$, respectively. The equilibrium bond distance $R_{\mathrm{e}}$ by the available all-electron relativistic calculations ${ }^{21}$ is $2.431 \AA$, and the corresponding percentage error is $1.25 \%$. The present $w_{\mathrm{e}} \chi_{\mathrm{e}}$ of the $\mathrm{X}^{1} \Sigma^{+}$state is $1.03 \mathrm{~cm}^{-1}$, which is slightly smaller than the previous 

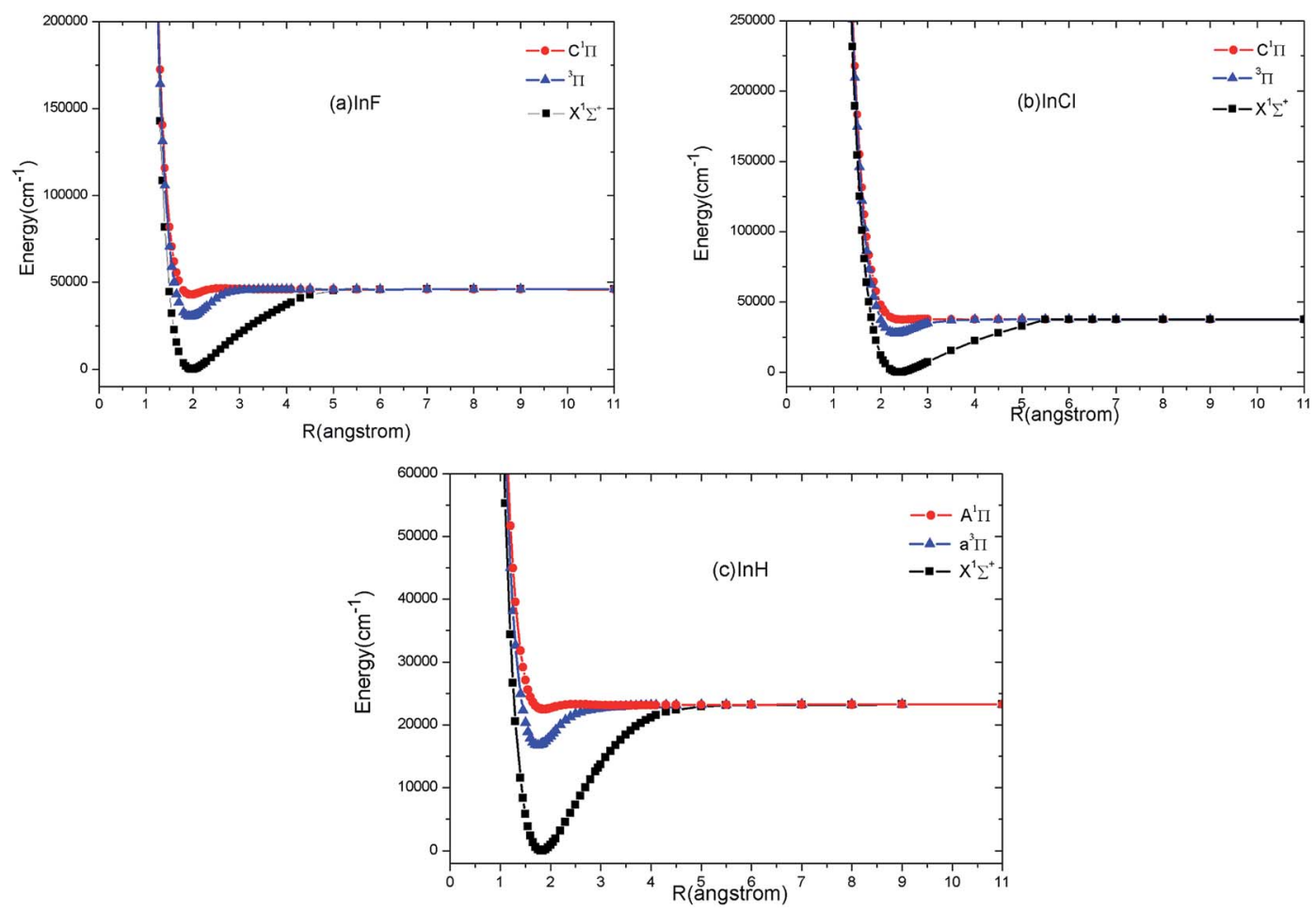

Fig. 1 Potential energy curves of the first three electronic states of $\operatorname{lnF}(\mathrm{a}), \operatorname{lnCl}(\mathrm{b})$ and $\ln \mathrm{H}$ (c) at the $\mathrm{MRCl}$ level of theory.

theoretical value $\mathrm{e}^{21} 1.38 \mathrm{~cm}^{-1}$. For the $\mathrm{C}^{1} \Pi$, our present results differences between the previous theoretical value ${ }^{21}$ and exper-

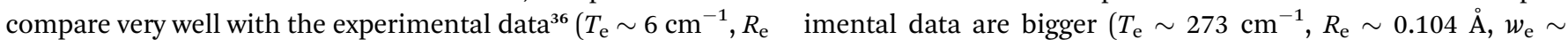
$\left.\sim 0.018 \AA, w_{\mathrm{e}} \sim 32.76 \mathrm{~cm}^{-1}, w_{\mathrm{e}} \chi_{\mathrm{e}} \sim 1.47 \mathrm{~cm}^{-1}\right)$. While the $70.40 \mathrm{~cm}^{-1}, w_{\mathrm{e}} \chi_{\mathrm{e}} \sim 9.27 \mathrm{~cm}^{-1}$ ). Whereas for the ${ }^{3} \Pi$, our

Table 1 Spectroscopic constants of $X^{1} \Sigma^{+}, C^{1} \Pi$ and ${ }^{3} \Pi$ states for $\operatorname{lnF} / \operatorname{lnCl}$ and $X^{1} \Sigma^{+}, A^{1} \Pi$ and $a^{3} \Pi$ states for $\operatorname{InH}$ calculated at the $M R C l$ level of theory

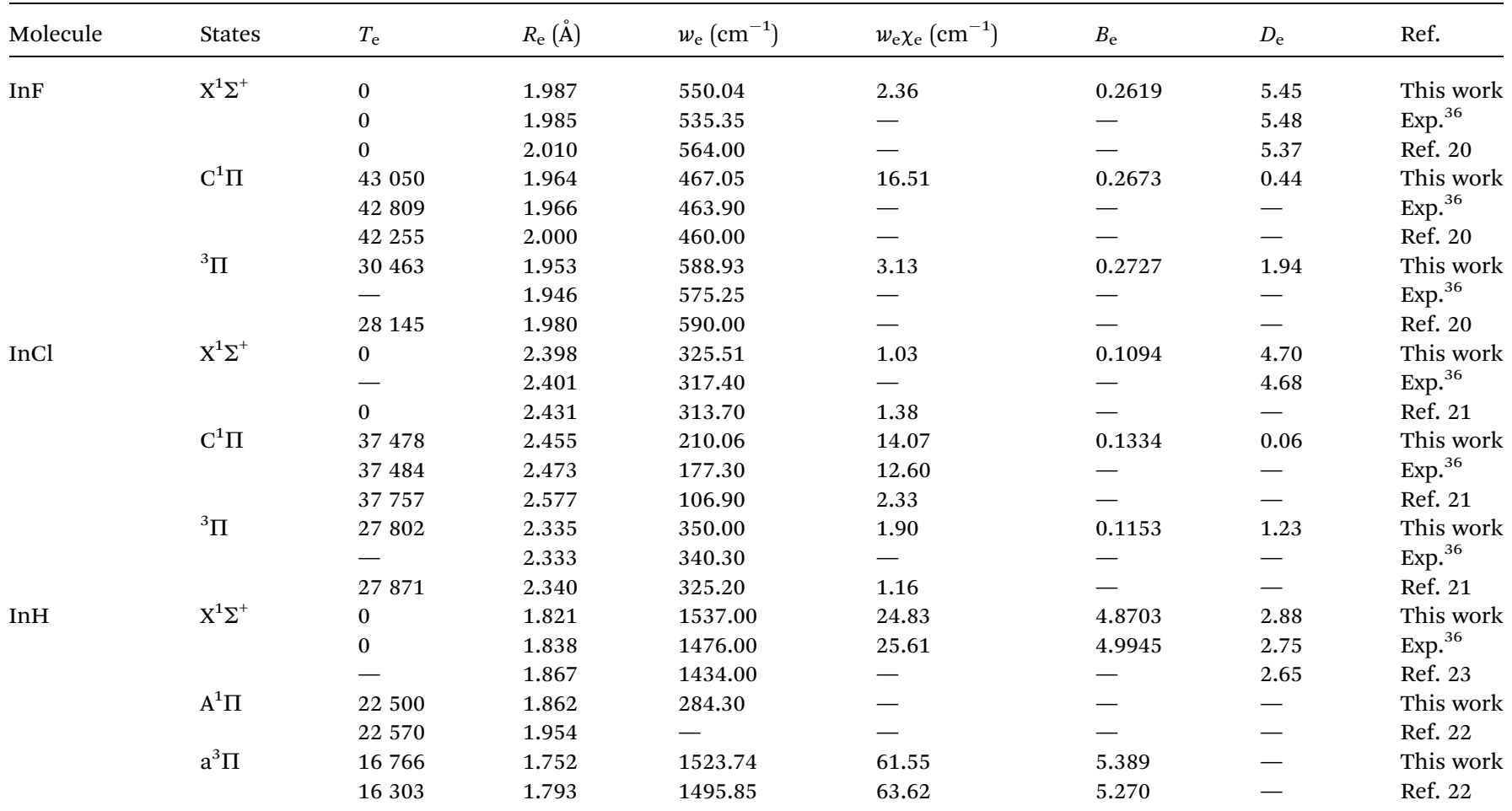




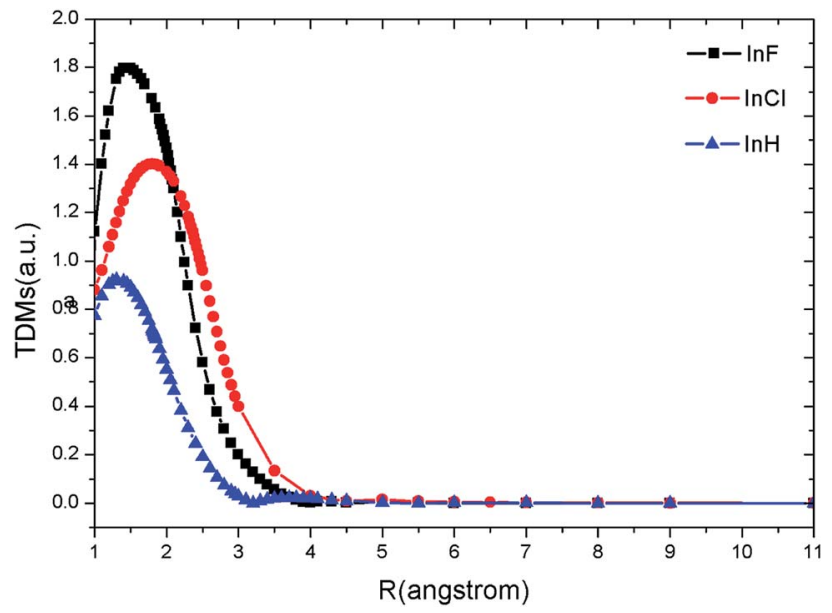

Fig. 2 TDMs for the $C^{1} \Pi \rightarrow X^{1} \Sigma^{+}$transitions of $\operatorname{lnF} / \operatorname{lnCl}$ and $A^{1} \Pi \rightarrow$ $X^{1} \Sigma^{+}$transitions of $\mathrm{InH}$ at $\mathrm{MRCl}$ level.

spectroscopic constants calculated at MRCI level are in reasonable agreement with the available all-electron relativistic calculations $^{21}\left(T_{\mathrm{e}} \sim 69 \mathrm{~cm}^{-1}, R_{\mathrm{e}} \sim 0.005 \AA, w_{\mathrm{e}} \sim 24.8 \mathrm{~cm}^{-1}, w_{\mathrm{e}} \chi_{\mathrm{e}}\right.$ $\left.\sim 0.74 \mathrm{~cm}^{-1}\right)$. In the case of $R_{\mathrm{e}}$ and $w_{\mathrm{e}}$, our results $(2.335 \AA$, $\left.350.0 \mathrm{~cm}^{-1}\right)$ are in good agreement with experiment ${ }^{36}(2.333 \AA$, $\left.340.3 \mathrm{~cm}^{-1}\right)$. The complete active space self-consistent field method (CASSCF) is used in both the previous work ${ }^{21}$ and our work. So the effects of the different active spaces (this work: 11,5,5,1) and (ref. 20: 6,3,3,1) on the spectroscopic constants of InCl can be seen from Table 1 . It is obvious that the calculated spectroscopic constants of InCl for active space $(11,5,5,1)$ are more close to the experimental values.

Concerning the $\mathrm{X}^{1} \Sigma^{+}$of InH, our calculated parameters $R_{\mathrm{e}}$, $w_{\mathrm{e}}, w_{\mathrm{e}} \chi_{\mathrm{e}}, B_{\mathrm{e}}$ and $D_{\mathrm{e}}$ are also in very good agreement with experiment, ${ }^{36}$ and the relative errors are $0.92 \%, 4.13 \%, 3.05 \%$, $2.49 \%$ and $4.73 \%$, respectively. While the percentage errors in $R_{\mathrm{e}}, w_{\mathrm{e}}$ and $D_{\mathrm{e}}$ given by Leininger et $a l^{23}$ are $1.58 \%, 2.85 \%$ and $3.64 \%$, respectively. For the $\mathrm{A}^{1} \Pi$ and $\mathrm{a}^{3} \Pi$ states of InH, Table 1 shows that our present results accord with existing theoretical calculations. $^{22,23}$ For example, the $\mathrm{A}^{1} \Pi$ and $\mathrm{a}^{3} \Pi$ states are located at $22500 \mathrm{~cm}^{-1}$ and $16766 \mathrm{~cm}^{-1}$ in our calculations, $22570 \mathrm{~cm}^{-1}$ and $16303 \mathrm{~cm}^{-1}$ were reported by Zhang et al. ${ }^{22}$

In summary, our present work yield spectroscopic parameters of InF, InCl and InH for the first three electronic states that agree well with previous experimental and theoretical results.

\subsection{TDM curves}

Because the transition ${ }^{3} \Pi \rightarrow \mathrm{X}^{1} \Sigma^{+}$is spin forbidden at spin-free level, we only obtain the TDMs of the ${ }^{1} \Pi \rightarrow \mathrm{X}^{1} \Sigma^{+}$transition.

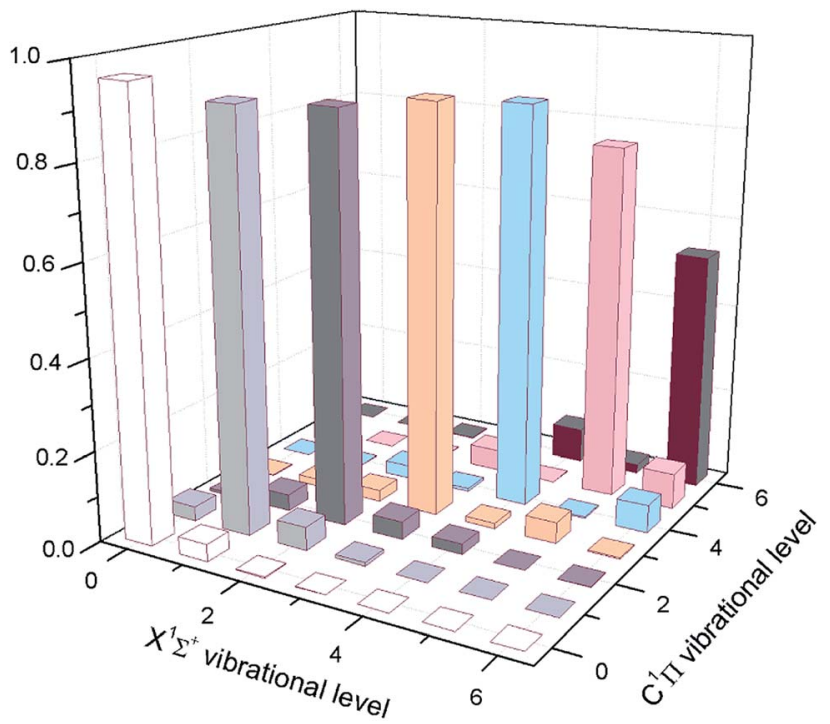

Fig. 3 The calculated FCFs of InF for the lowest vibrational levels of the cooling transition $C^{1} \Pi \rightarrow X^{1} \Sigma^{+}$.

Fig. 2 shows the computed TDMs of the dipole-allowed transitions $\mathrm{C}^{1} \Pi \rightarrow \mathrm{X}^{1} \Sigma^{+}$for $\mathrm{InF} / \mathrm{InCl}$ and $\mathrm{A}^{1} \Pi \rightarrow \mathrm{X}^{1} \Sigma^{+}$for InH as functions of the internuclear distance. We note that the ${ }^{1} \Pi \rightarrow$ $\mathrm{X}^{1} \Sigma^{+}$transitions for InF, InCl and InH are strong. As seen in Fig. 2, the TDM curves of InF, InCl and InH demonstrate similar behavior. The magnitude gradually increases as internuclear distance $R$ increases, reaches a maximum (InF: 1.80 a.u., InCl: 1.40 a.u., InH: 0.92 a.u.), and drops thereafter. The TDMs of InF, InCl and InH all trend to zero at around $4.0 \AA$, $3.5 \AA$ and $3.0 \AA$, respectively.

\subsection{FCFs, spontaneous radiative lifetime and radiative width}

3.3.1 InF molecule. The potential laser cooling candidates must have highly diagonal Franck-Condon factors (FCFs). The calculated FCF data of the $\mathrm{C}^{1} \Pi \rightarrow \mathrm{X}^{1} \Sigma^{+}$transition have been tabulated in Table 2. It is clear that InF has highly diagonal FCFs $f_{\nu^{\prime} \nu}\left(f_{00}=0.961\right.$ and $\left.f_{11}=0.909\right)$ for the $\mathrm{C}^{1} \Pi\left(\nu^{\prime}\right) \rightarrow \mathrm{X}^{1} \Sigma^{+}(\nu)$ transition. Most FCFs of experimental cooling molecules are greater than 0.9 , as listed in the previous literature. ${ }^{6-10}$ The predicted $f_{00}$ of 0.961 for InF is greater than 0.9 , thus it is sufficiently large to be potentially viable for cooling. Of course, a larger FCF has significant benefits for limiting the number of lasers required to keep the molecule in a quasi-closed-loop cooling cycle. To visually demonstrate the distributions of FCFs $f_{\nu^{\prime} \nu}$ for the different vibrational states of the $\mathrm{C}^{1} \Pi\left(\nu^{\prime}\right) \rightarrow$ $\mathrm{X}^{1} \Sigma^{+}(\nu)$ transition, we have plotted the cooling transition

Table 2 The calculated FCFs $f_{\nu^{\prime} \nu}$ for $C^{1} \Pi\left(\nu^{\prime}\right) \rightarrow X^{1} \Sigma^{+}(\nu)$ and ${ }^{3} \Pi\left(\nu^{\prime}\right) \rightarrow X^{1} \Sigma^{+}(\nu)$ transitions of $\operatorname{InF}$

\begin{tabular}{llllr}
\hline Molecule & Transition & $f_{00}, f_{10}$ & $f_{01}, f_{11}$ & $f_{02}, f_{12}$ \\
\hline InF & $\mathrm{C}^{1} \Pi \rightarrow \mathrm{X}^{1} \Sigma^{+}$ & $0.9606,0.0377$ & $0.0326,0.9085$ & $0.0062,0.0369$ \\
& ${ }^{3} \Pi \rightarrow \mathrm{X}^{1} \Sigma^{+}$ & $0.8068,0.1788$ & $0.1670,0.4997$ & $0.0231,0.2542$
\end{tabular}


Table 3 Estimated radiative lifetimes (ns) and radiative width $\left(\mathrm{cm}^{-1}\right.$ ) (in italics) (theoretical values obtained in brackets)

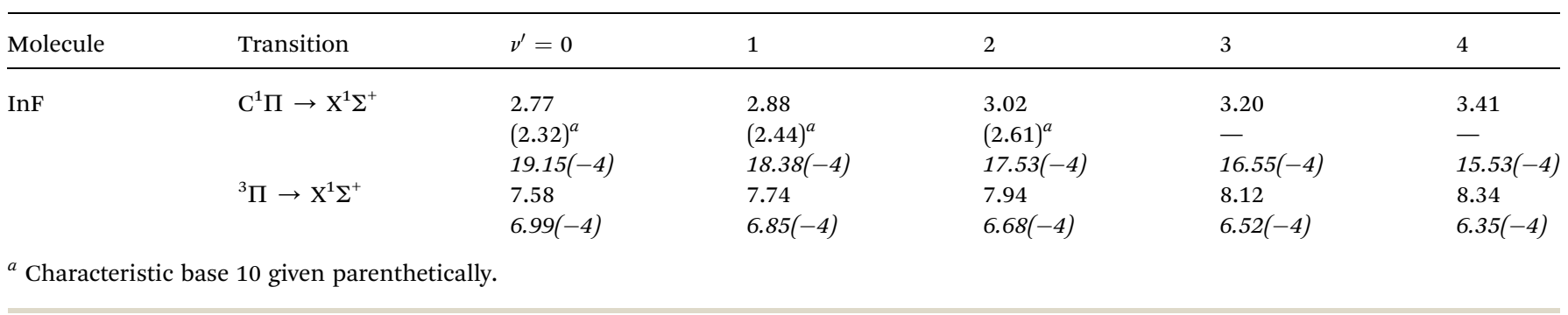

$\mathrm{C}^{1} \Pi\left(\nu^{\prime}\right) \rightarrow \mathrm{X}^{1} \Sigma^{+}(\nu)$ between $0 \leqq \nu^{\prime} \leqq 6$ and $0 \leqq \nu \leqq 6$ in Fig. 3 . The FCFs for the ${ }^{3} \Pi \rightarrow \mathrm{X}^{1} \Sigma^{+}$transition are also presented in Table 2 . The ${ }^{3} \Pi \rightarrow \mathrm{X}^{1} \Sigma^{+}$transition also have large diagonal FCFs, although the calculated $f_{00}$ of 0.807 for the ${ }^{3} \Pi \rightarrow \mathrm{X}^{1} \Sigma^{+}$ transition is smaller than that predicted for the $\mathrm{C}^{1} \Pi \rightarrow \mathrm{X}^{1} \Sigma^{+}$ transition $\left(f_{\text {oo }}=0.961\right)$.

Except for large FCFs, the potential laser cooling candidates must also have sufficiently short lifetimes. In Table 3, we have reported the computed lifetimes for the $\mathrm{C}^{1} \Pi \rightarrow \mathrm{X}^{1} \Sigma^{+}$transition. Our computed lifetimes can be compared with those of the results predicted by Banerjee et al. ${ }^{20}$ The radiative lifetimes of the $\mathrm{C}^{1} \Pi \rightarrow \mathrm{X}^{1} \Sigma^{+}$transition are computed to be 2.77-3.41 ns for the first five vibrational levels of InF, which shows that the $C^{1} \Pi$ of InF is a rather short lifetime state. At the same time, the radiative width for the $\mathrm{C}^{1} \Pi \rightarrow \mathrm{X}^{1} \Sigma^{+}$transition are computed to be $19.15-15.53 \mathrm{~cm}^{-1}$ for the first five vibrational levels of InF. The radiative lifetimes (7.58-8.34 ns) of the ${ }^{3} \Pi$ are a little longer lived than these of the electronic state $C^{1} \Pi$, which shows that the ${ }^{3} \Pi$ of InF is also a rather short lifetime state. As shown in Table 3, the radiative lifetimes show a slight increase with increasing vibrational level, and the radiative widths show a slight decrease with increasing vibrational level.

3.3.2 InCl molecule. For the $\mathrm{C}^{1} \Pi\left(\nu^{\prime}\right) \rightarrow \mathrm{X}^{1} \Sigma^{+}(\nu)$ transition of InCl, the diagonal term $f_{11}(0.07)$ is obtained, and the offdiagonal term $f_{10}(0.29), f_{12}(0.16), f_{13}(0.18)$ are also calculated (see Table 4). For the ${ }^{3} \Pi \rightarrow \mathrm{X}^{1} \Sigma^{+}$transition of InCl, off-diagonal term $f_{10}(0.29), f_{12}(0.39), f_{13}(0.15)$ are larger than the diagonal term $f_{11}(0.14)$ (see Table 4$)$. It is clear that InCl does not have highly diagonal FCFs for the $\mathrm{C}^{1} \Pi \rightarrow \mathrm{X}^{1} \Sigma^{+}$and ${ }^{3} \Pi \rightarrow \mathrm{X}^{1} \Sigma^{+}$ transitions. Besides, the present FCFs $f_{\text {oo }}(0.61)$ for the $\mathrm{C}^{1} \Pi \rightarrow$ $\mathrm{X}^{1} \Sigma^{+}$transition and $f_{00}(0.60)$ for the ${ }^{3} \Pi \rightarrow \mathrm{X}^{1} \Sigma^{+}$transition are not large enough to be potentially viable for cooling. The relative probabilities from $\mathrm{C}^{1} \Pi\left(\nu^{\prime}=0\right)$ to $\mathrm{X}^{1} \Sigma^{+}(\nu)$ are governed by the FCFs which are approximately $61 \%$ for $\nu=0,23 \%$ for $\nu=1,10 \%$ for $\nu=2,4 \%$ for $\nu=3,1.5 \%$ for $\nu=4,0.5 \%$ for $\nu=5,0.2 \%$ for $\nu$ $=6,0.07 \%$ for $\nu=7$, and negligibly small for all $\nu>7$. To limit the inefficiency, or loss of InCl molecules in the cooling cycle, eight cooling lasers are required. Experimentally, cycling transitions requiring one or two repump lasers are common in atomic systems. Here the number of lasers required is not practical. There are similarities to the laser cooling of $\mathrm{InCl}$ on the ${ }^{3} \Pi \rightarrow \mathrm{X}^{1} \Sigma^{+}$transition. So we identify that $\mathrm{InCl}$ is not a potential laser-cooling candidate. Thus the radiative lifetimes and diode laser excitation wavelengths of $\mathrm{InCl}$ do not need to be discussed in the following section.

3.3.3 InH molecule. As listed in Table 5 , the $A^{1} \Pi$ state is not suitable for laser cooling because of the off-diagonal FCFs and the small diagonal FCF $f_{00}(0.790)$ for the $\mathrm{A}^{1} \Pi \rightarrow \mathrm{X}^{1} \Sigma^{+}$transition. Fig. 4 shows the FCFs of the $\mathrm{a}^{3} \Pi \rightarrow \mathrm{X}^{1} \Sigma^{+}$transition are highly diagonal. The present FCF of 0.918 for the $\mathrm{a}^{3} \Pi \rightarrow \mathrm{X}^{1} \Sigma^{+}$ transition of InH is still large enough for laser cooling. However, this $\mathrm{a}^{3} \Pi \rightarrow \mathrm{X}^{1} \Sigma^{+}$transition is very weak because it is spinforbidden. Because of the poor linestrength of the $\mathrm{a}^{3} \Pi \rightarrow$ $\mathrm{X}^{1} \Sigma^{+}$transition, the InH molecule must have been precooled using the $\mathrm{A}^{1} \Pi \rightarrow \mathrm{X}^{1} \Sigma^{+}$transition before the $\mathrm{a}^{3} \Pi \rightarrow \mathrm{X}^{1} \Sigma^{+}$ transition could be adopted for laser cooling of InH. It is similar to the laser cooling of $\mathrm{Ca}$, where cooling on the weak ${ }^{1} \mathrm{~S} \rightarrow{ }^{1} \mathrm{P}$ transition must followed by the strong ${ }^{1} \mathrm{~S} \rightarrow{ }^{3} \mathrm{P}$ transition. The problem with the laser cooling scheme using the $\mathrm{a}^{3} \Pi \rightarrow \mathrm{X}^{1} \Sigma^{+}$ transition is that the InH molecule could not be precooled using the $\mathrm{A}^{1} \Pi \rightarrow \mathrm{X}^{1} \Sigma^{+}$transition. So InH is not a promising lasercooling candidate. Thus the radiative lifetimes and diode laser excitation wavelengths of InH do not need to be discussed in the following section.

\subsection{Laser cooling scheme}

We have established the laser cooling schemes for InF, and Fig. 5 shows the proposed scheme. The corresponding computed radiative wavelengths are collected in Table 6. For InF, the main cycle is the $\mathrm{X}^{1} \Sigma^{+}(\nu=0) \rightarrow \mathrm{C}^{1} \Pi\left(\nu^{\prime}=0\right)$ transition at wavelength $\lambda_{00}=232.4 \mathrm{~nm}$. As shown in Fig. 5(a), there is a small probability of decay to the $\mathrm{X}^{1} \Sigma^{+}(\nu=1)$ state $(\approx 3 \%)$, an even smaller probability of decay to the $\mathrm{X}^{1} \Sigma^{+}(\nu=2)$ state $(\approx 0.6 \%)$, and a negligible probability of decay to the $\mathrm{X}^{1} \Sigma^{+}(\nu \geq 3)$

Table 4 The calculated FCFs $f_{\nu^{\prime} \nu}$ for $C^{1} \Pi\left(\nu^{\prime}\right) \rightarrow X^{1} \Sigma^{+}(\nu)$ and ${ }^{3} \Pi\left(\nu^{\prime}\right) \rightarrow X^{1} \Sigma^{+}(\nu)$ transitions of InCl

\begin{tabular}{llllr}
\hline Molecule & Transition & $f_{00}, f_{10}$ & $f_{01}, f_{11}$ & $f_{02}, f_{12}$ \\
\hline InCl & $\mathrm{C}^{1} \Pi \rightarrow \mathrm{X}^{1} \Sigma^{+}$ & $0.6079,0.2912$ & $0.2293,0.0716$ & $0.1010,0.1620$ \\
& ${ }^{3} \Pi \rightarrow \mathrm{X}^{1} \Sigma^{+}$ & $0.5957,0.2907$ & $0.3282,0.1437$ & $0.0691,0.3913$
\end{tabular}


Table 5 The calculated FCFs $f_{\nu^{\prime} \nu}$ for $\mathrm{A}^{1} \Pi\left(\nu^{\prime}\right) \rightarrow \mathrm{X}^{1} \Sigma^{+}(\nu)$ and $a^{3} \Pi\left(\nu^{\prime}\right) \rightarrow \mathrm{X}^{1} \Sigma^{+}(\nu)$ transitions of $\operatorname{InCl}$

\begin{tabular}{lllll}
\hline Molecule & Transition & $f_{00}, f_{10}$ & $f_{01}, f_{11}$ & $f_{02}, f_{12}$ \\
\hline InH & $\mathrm{A}^{1} \Pi \rightarrow \mathrm{X}^{1} \Sigma^{+}$ & $0.7897,0.0573$ & $0.1172,0.0169$ & $0.0604,0.0372$ \\
& $\mathrm{a}^{3} \Pi \rightarrow \mathrm{X}^{1} \Sigma^{+}$ & $0.9176,0.0672$ & $0.0809,0.8217$ & $0.0012,0.0969$
\end{tabular}

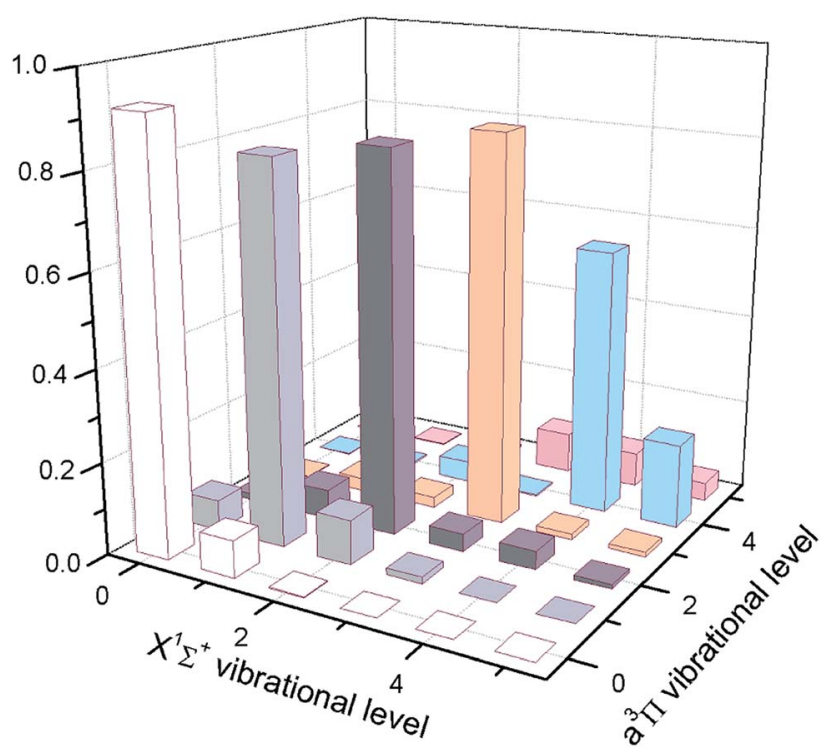

Fig. 4 The calculated FCFs of InH for the lowest vibrational levels of the cooling transition $a^{3} \Pi \rightarrow X^{1} \Sigma^{+}$.

state $\left(<10^{-4}\right)$. To enhance the cooling effect, we add the $\mathrm{X}^{1} \Sigma^{+}(\nu=$ 1) $\rightarrow \mathrm{C}^{1} \Pi\left(\nu^{\prime}=0\right)$ transition as the first vibrational pump and the $\mathrm{X}^{1} \Sigma^{+}(\nu=2) \rightarrow \mathrm{C}^{1} \Pi\left(\nu^{\prime}=1\right)$ transition for the second pump. With this scheme, two repumping lasers $\lambda_{10}=229.8 \mathrm{~nm}$ and $\lambda_{21}$ $=230.2 \mathrm{~nm}$ are required. The required cooling wavelengths of $\lambda_{00}=232.4 \mathrm{~nm}, \lambda_{10}=229.8 \mathrm{~nm}$ and $\lambda_{21}=230.2 \mathrm{~nm}$ can be generated by a frequency quadrupled Ti:sapphire laser (189-235 $\mathrm{nm}$ ). One problem with the above laser cooling scheme, is the presence of the intervening ${ }^{3} \Pi$ state between the two states $\left(\mathrm{X}^{1} \Sigma^{+}\right.$and $\left.\mathrm{C}^{1} \Pi\right)$. Notable, the ${ }^{3} \Pi \rightarrow \mathrm{X}^{1} \Sigma^{+}$transition also have
Table 6 The calculated wavelength $\lambda_{\nu^{\prime} \nu}$

\begin{tabular}{llllll}
\hline Molecule & $\lambda_{00}(\mathrm{~nm})$ & $\lambda_{10}(\mathrm{~nm})$ & $\lambda_{21}(\mathrm{~nm})$ & $\lambda_{31}(\mathrm{~nm})$ & Transition \\
\hline InF & 232.4 & 229.8 & 230.2 & 227.8 & $\mathrm{C}^{1} \Pi \rightarrow \mathrm{X}^{1} \Sigma^{+}$ \\
& 328.1 & 321.9 & 321.6 & 315.8 & ${ }^{3} \Pi \rightarrow \mathrm{X}^{1} \Sigma^{+}$ \\
InH & 596.9 & 549.5 & 556.3 & 525.0 & $\mathrm{a}^{3} \Pi \rightarrow \mathrm{X}^{1} \Sigma^{+}$
\end{tabular}

large diagonal FCFs and short lifetimes. Thus, this state ${ }^{3} \Pi$ could also be another option for laser cooling. We propose a four laser cyclic system involving the $\nu=0-3$ of the $\mathrm{X}^{1} \Sigma^{+}$state and $\nu^{\prime}=0,1$ of the ${ }^{3} \Pi$ state. The main cycling laser at the wavelength of $328.1 \mathrm{~nm}$ can drive the $\mathrm{X}^{1} \Sigma^{+}(\nu=0) \rightarrow{ }^{3} \Pi\left(\nu^{\prime}=0\right)$ transition. Similar to the case in Fig. 5(a), a pair of repumping lasers with $\lambda_{10}=321.9 \mathrm{~nm}$ and $\lambda_{21}=321.6 \mathrm{~nm}$ are required to enhance the cooling effect. A third repumping laser with $\lambda_{31}=$ $315.8 \mathrm{~nm}$ may be required on this scheme due to the nonnegligible $\nu^{\prime}=1 \rightarrow \nu=3$ transition (FCF $\left.=0.0565\right)$. The required cooling wavelengths on this scheme are located in the ultraviolet light (UVA) range (400-320 (or 315) nm). There are similarities here to the laser cooling of AlF. Wells and Lane ${ }^{15}$ have studied on laser cooling of AlF molecules, and the $\mathrm{X}^{1} \Sigma^{+} \rightarrow$ $\mathrm{C}^{1} \Pi$ and $\mathrm{X}^{1} \Sigma^{+} \rightarrow{ }^{3} \Pi$ transitions of AlF had strongly FCFs. So for InF, the cooling on a strong $\mathrm{C}^{1} \Pi \rightarrow \mathrm{X}^{1} \Sigma^{+}$transition may be followed by further cooling on the $\mathrm{X}^{1} \Sigma^{+} \rightarrow{ }^{3} \Pi$ transition, which is similar to the laser cooling of AlF. The relatively long lifetimes of the ${ }^{3} \Pi$ state can be exploited to reach a much lower Doppler temperature than possible on the $\mathrm{X}^{1} \Sigma^{+} \rightarrow \mathrm{C}^{1} \Pi$ transition.

Compared with other potential laser-cooling candidates, diagonal FCFs $f_{00}$ of $\operatorname{InF}\left(f_{00}=0.961\right)$ is slightly larger than that predicted for $\operatorname{BeF}\left(f_{00}=0.897\right)$, LiRb $\left(f_{00}=0.872\right)$, InH $\left(f_{00}=\right.$ $0.918)$ and $\mathrm{KRb}\left(f_{\mathrm{oo}}=0.947\right)$. Experimentally, $\mathrm{KRb}$ is a typical
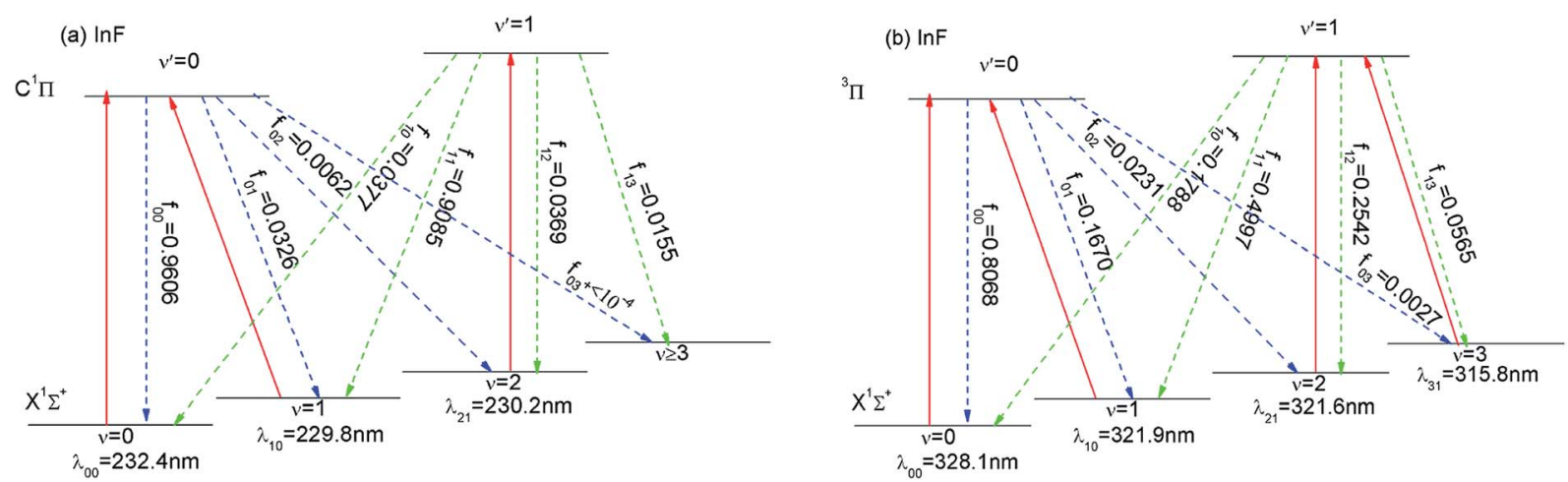

Fig. 5 Proposed laser cooling schemes for InF using (a) the $C^{1} \Pi\left(\nu^{\prime}\right) \rightarrow X^{1} \Sigma^{+}(\nu)$ (solid red) and (b) the ${ }^{3} \Pi\left(\nu^{\prime}\right) \rightarrow X^{1} \Sigma^{+}(\nu)$ (solid red) transition. The decay pathways with calculated $f_{\nu^{\prime} \nu}$ are shown as dotted line. 
laser-cooled molecule. Radiative lifetime $\tau\left(\nu^{\prime}=0\right)=2.8 \mathrm{~ns}$ of InF is shorter than the one of BeF (7.9 ns), LiRb (102.6 ns), InH (205.7 ns) and $\mathrm{KRb}$ (229.3 ns). A shorter $\tau$ of the transition could produce a strong Doppler force, which will produce rapid laser cooling. We have identified InF is a more promising lasercooling candidate. Not only it has bigger FCFs and shorter radiative lifetime, it also appears to attain a lower Doppler temperature on the $\mathrm{X}^{1} \Sigma^{+} \rightarrow \mathrm{C}^{1} \Pi$ transition followed by the weak $\mathrm{X}^{1} \Sigma^{+} \rightarrow{ }^{3} \Pi$ transition. Generally, it is true that the Doppler-limit temperature is inversely proportional to the excited state lifetime. As shown in Table 3, the lifetime (7.58 ns) of the ${ }^{3} \Pi$ state is about three times larger than that of the $C^{1} \Pi$ state. However, the lifetime (7.58 ns) of InF is still shorter than that of $\mathrm{KRb}$ ( $229.3 \mathrm{~ns}$ ). The short lifetime is sufficient to produce large spontaneous scattering forces.

\section{Conclusions}

The PECs of $\mathrm{X}^{1} \Sigma^{+},{ }^{1} \Pi$ and ${ }^{3} \Pi$ states of $\mathrm{InF}, \mathrm{InCl}$ and $\mathrm{InH}$ are acquired at the MRCI level. Our present work yield spectroscopic constants $\left(R_{\mathrm{e}}, D_{\mathrm{e}}, w_{\mathrm{e}}, w_{\mathrm{e}} \chi_{\mathrm{e}}, B_{\mathrm{e}}, T_{\mathrm{e}}\right)$ of InF, InCl and InH for the first three electronic states that agree well with previous experimental and theoretical results. The TDMs of the dipoleallowed transitions $\mathrm{C}^{1} \Pi \rightarrow \mathrm{X}^{1} \Sigma^{+}$for $\mathrm{InF} / \mathrm{InCl}$ and $\mathrm{A}^{1} \Pi \rightarrow$ $\mathrm{X}^{1} \Sigma^{+}$for InH are obtained. Using the FCFs and radiative lifetimes obtained, we have identified InF is a promising candidate for laser cooling. Because of its off-diagonal FCFs, InCl is not a potential laser-cooling candidate. For $\mathrm{InH}$, the $\mathrm{A}^{1} \Pi$ state has off-diagonal FCFs and the FCFs of the $\mathrm{a}^{3} \Pi \rightarrow \mathrm{X}^{1} \Sigma^{+}$transition are highly diagonal. However, this $\mathrm{a}^{3} \Pi \rightarrow \mathrm{X}^{1} \Sigma^{+}$transition is very weak because it is spin-forbidden. The problem with the laser cooling scheme using the $\mathrm{a}^{3} \Pi \rightarrow \mathrm{X}^{1} \Sigma^{+}$transition is that the InH molecule could not be precooled using the $\mathrm{A}^{1} \Pi \rightarrow \mathrm{X}^{1} \Sigma^{+}$transition. So InH is not a promising laser-cooling candidate. The proposed laser cooling schemes of InF that drive the $\mathrm{X}^{1} \Sigma^{+} \rightarrow$ $\mathrm{C}^{1} \Pi$ transition use three laser wavelength. Furthermore, the ${ }^{3} \Pi$ $\rightarrow \mathrm{X}^{1} \Sigma^{+}$transition of $\mathrm{InF}$ is also strongly diagonal and the lifetimes of the excited ${ }^{3} \Pi$ state of $\mathrm{InF}$ is relatively long. So for InF, the $\mathrm{X}^{1} \Sigma^{+} \rightarrow \mathrm{C}^{1} \Pi$ transition may be followed by the weak $\mathrm{X}^{1} \Sigma^{+} \rightarrow{ }^{3} \Pi$ transition to obtain a lower Doppler temperature. All of these three required laser-cooling wavelengths are in the visible range.

\section{Conflicts of interest}

There are no conflicts to declare.

\section{Acknowledgements}

This work was supported by the National Natural Science Foundation of China (Grant No. 11704052).

\section{References}

1 A. Micheli, G. Brennen and P. Zoller, Nat. Phys., 2006, 2, 341. 2 R. V. Krems, Phys. Chem. Chem. Phys., 2008, 10, 4079.
3 V. V. Flambaum and M. G. Kozlov, Phys. Rev. Lett., 2007, 99, 150801.

4 T. A. Isaev, S. Hoekstra and R. Berger, Phys. Rev. A: At., Mol., Opt. Phys., 2010, 82, 052521.

5 J. J. Hudson, D. M. Kara, I. J. Smallman, B. E. Sauer, M. R. Tarbutt and E. A. Hinds, Nature, 2011, 473, 493.

6 E. S. Shuman, J. F. Barry and D. DeMille, Nature, 2010, 467, 820.

7 J. F. Barry, D. J. McCarron, E. B. Norrgard, M. H. Steinecker and D. DeMille, Nature, 2014, 512, 286.

8 M. T. Hummon, M. Yeo, B. K. Stuhl, A. L. Collopy, Y. Xia and J. Ye, Phys. Rev. Lett., 2013, 110, 143001.

9 V. Zhelyazkova, A. Cournol, T. E. Wall, A. Matsushima, J. J. Hudson, E. A. Hinds, M. R. Tarbutt and B. E. Sauer, Phys. Rev. A: At., Mol., Opt. Phys., 2014, 89, 053416.

10 J. Kobayashi, K. Aikawa, K. Oasa and S. Inouye, Phys. Rev. A: At., Mol., Opt. Phys., 2014, 89, 021401(R).

11 I. C. Lane, Phys. Chem. Chem. Phys., 2012, 14, 15078.

12 S. Y. Kang, Y. F. Gao, F. G. Kuang, T. Gao, J. G. Du and G. Jiang, Phys. Rev. A: At., Mol., Opt. Phys., 2015, 91, 042511.

13 Y. F. Gao and T. Gao, Phys. Rev. A: At., Mol., Opt. Phys., 2014, 90, 052506.

14 Y. You, C. L. Yang, M. S. Wang, X. G. Ma and W. W. Liu, Phys. Rev. A: At., Mol., Opt. Phys., 2015, 92, 032502.

15 N. Wells and I. C. Lane, Phys. Chem. Chem. Phys., 2011, 13, 19018.

16 R. Yang, B. Tang and T. Gao, Chin. Phys. B, 2016, 4, 043101.

17 R. Yang, Y. F. Gao, B. Tang and T. Gao, Phys. Chem. Chem. Phys., 2015, 17, 1900.

18 C. L. Li, Y. C. Li, Z. H. Ji, X. B. Qiu, Y. Z. Lai, J. L. Wei, Y. T. Zhao, L. H. Deng, Y. Q. Chen and J. J. Liu, Phys. Rev. A, 2018, 97, 062501.

19 Y. F. Gao and T. Gao, Phys. Chem. Chem. Phys., 2015, 17, 10830.

20 A. Banerjee, A. Pramanik and K. K. Das, Chem. Phys. Lett., 2006, 429, 62.

21 W. L. Zou, M. R. Lin, X. Z. Yang and B. Z. Zhang, J. Chem. Phys., 2003, 119, 3721.

22 Y. G. Zhang, H. Zhang and G. Dou, Chin. Phys. B, 2017, 9, 093101.

23 T. Leininger, A. Nicklass, H. Stoll, M. Dolg and P. Schwerdtfeger, J. Chem. Phys., 1996, 105, 1052.

24 H.-J. Werner, P. J. Knowles, R. Lindh, F. R. Manby, M. Schütz, et al., MOLPRO, is a package of ab initio programs, version 2009.1, see http://www.molpro.net.

25 H.-J. Werner and P. J. Knowles, J. Chem. Phys., 1985, 82, 5053.

26 P. J. Knowles and H.-J. Werner, Chem. Phys. Lett., 1985, 115, 259.

27 H.-J. Werner and P. J. Knowles, J. Chem. Phys., 1988, 89, 5803. 28 P. J. Knowles and H.-J. Werner, Chem. Phys. Lett., 1988, 145, 514.

29 S. R. Laughoff and E. R. Davidson, Int. J. Quantum Chem., 1974, 8, 61.

30 N. Douglas and N. M. Kroll, Ann. Phys., 1974, 82, 89.

31 B. A. Hess, Phys. Rev. A: At., Mol., Opt. Phys., 1986, 33, 3742.

32 R. J. Le Roy, LEVEL 8.0, A Computer Program for Solving the Radial Schrödinger Equation for Bound and Quasibound 
Levels, CPRR-663, University of Waterloo, Waterloo, ON, 35 A. K. Wilson, D. E. Woon, K. A. Peterson and T. H. Dunning 2007. Jr, J. Chem. Phys., 1999, 110, 7667.

33 I. S. Lim, H. Stoll and P. Schwerdtfeger, J. Chem. Phys., 2006, 36 K. P. Huber and G. Herzberg, Molecular Spectra and 124, 034107.

34 T. H. Dunning Jr, J. Chem. Phys., 1989, 90, 1007. Molecular Structure IV: Constants of Diatomic Molecules, Van Nostrand, New York, 1979. 